\title{
Editorial
}

Welcome to the first edition of the World's Poultry Science Journal for 2010. I trust all our members are enjoying a productive and interesting new year. However, as always there are challenges and opportunities ahead within the agricultural sectors of the world, including our own poultry world. The economic climate continues to be difficult, and, even though there has been media discussion of an upturn, it looks as though the situation will continue for the next 12 months at least, and maybe longer in some regions. Many of the developed countries affected continue to have problems within the poultry production sector with major losses being reported, especially in the USA. Globally, however agriculture is possibly less affected compared to other businesses. Let's hope this continues to be the case. Perhaps the major changes and efficiencies made in the last 15 years or so within poultry will be the saving grace of the industry is such difficult times. Of course, people will always need to eat, and poultry meat and eggs continue to be a prime source of essential nutrients and cost-effective protein for the human population.

Within the press we still have the usual issues rumbling on - concerns about using genetically modified feed materials, the move towards higher welfare systems especially for laying hens, poultry as a vector for disease, the carbon footprint of production and pollution. However poultry science is devoting much effort and funding to solving these issues, and meat and eggs can only benefit as a result of being viewed as a health form of food. Hopefully the costs of implementing some of these changes will not cause further economic problems, and buyers and consumers will recognise the need to meet any extra costs. In addition, many areas have experienced severe weather in the last few months which will hopefully not affect the feed and meat industry unduly - although some Asian countries have been affected to the extent they have increased poultry imports.

On the positive side, white meat often becomes the choice of consumers with shrinking purses, which bodes well for our industry. We also have the usual list of excellent WPSA-linked conferences, seminars and events to look forward to in the coming year. These are always highly worthwhile supporting, whether you are a researcher, academic or commercial poultry scientist, as it is an excellent way of getting up to date with the latest ideas and knowledge in an efficient manner, as well as making the most of networking opportunities. The current issue contains the bi-annual INFPD papers, which includes information on diseases important within developing countries. The WPSJ continues to be very proud of its role with the FAO in hosting these papers please keep sending them in - the more variety within the topics addressed the better. Likewise I am very pleased to say that we are getting more unsolicited papers than ever before. However, I would like to reiterate that we only accept review papers - please send any single experiments to our sister journal British Poultry Science. Regarding sending in papers, we also have the exciting announcement that anyone with a suitable paper for the journal will, from now on, be submitting on-line. Simply go onto the WPSA website and follow the instructions and links.

\section{Lucy Tucker}

Editor 